\title{
Surface structure and anion order of the oxynitride $\mathrm{LaTiO}_{2} \mathrm{~N}^{\dagger}$
}

\author{
Silviya Ninova and Ulrich Aschauer*
}

\begin{abstract}
Oxynitrides are promising materials for water splitting under visible light. Members of this class of semiconductors that crystallise in the perovskite structure are often characterised by $\mathrm{O} / \mathrm{N}$ disorder, while some studies observe 2D cischain ordering of the M-N-M bonds in the bulk. Despite the fact that the surface structure and composition is expected to have a significant influence on the surface chemistry and therefore the photocatalytic activity, little is known about the $\mathrm{O} / \mathrm{N}$ arrangement at surfaces of these materials. In the present study, we investigate the surface structure of $\mathrm{LaTiO}_{2} \mathrm{~N}$, a particularly promising candidate for water splitting, using density functional theory (DFT) calculations. Based on slab calculations with different anion order we find that the $\mathrm{N}$ atoms prefer to form trans-chains at the (001) surface, as opposed to the bulk. This is governed by the electrostatic stability that is optimal for alternating charge-neutral (LaN)- $\left(\mathrm{TiO}_{2}\right)$ atomic layers. We show that polar surfaces that do not fulfil this requirement will electronically or structurally reconstruct. Our results predict that in contact with vacuum, the $\mathrm{LaTiO}_{2} \mathrm{~N}$ (001) surface will preferentially be LaN-terminated.
\end{abstract}

\section{Introduction}

Oxynitrides constitute a class of semiconductors that have recently attracted increased attention for their ability to split water under visible light ${ }^{1-5}$. These materials can be regarded as derived from pure oxides via partial anion substitution, which affects their properties. In particular, the substitution with nitrogen, a less electronegative element than oxygen, leads to a reduction of the band gap, so that oxynitrides absorb a larger portion of the solar spectrum compared to pure oxides. This, however, comes at the price of reduced stability in such a mixed compound ${ }^{6}$.

A significant fraction of oxynitrides occur in the perovskite crystal structure. Similarly to perovskite oxides, perovskite oxynitrides commonly exhibit structural instabilities leading to deviations from the perfect cubic cell ${ }^{7}$. In addition, the anion sites can be occupied by either $\mathrm{O}$ or $\mathrm{N}$ ions, further influencing the crystal structure. Much effort has been dedicated to discriminate the $\mathrm{O} / \mathrm{N}$ arrangement in the bulk of a variety of oxynitride compounds, both experimentally and theoretically ${ }^{7,8}$. Many perovskite oxynitrides, such as $\mathrm{SrNbO}_{2} \mathrm{~N}, \mathrm{SrTaO}_{2} \mathrm{~N}^{9}$ and $\mathrm{BaTaO}_{2} \mathrm{~N}^{10}$ exhibit only partial ordering, while others

${ }^{*}$ University of Bern, Department of Chemistry and Biochemistry, Bern, Switzerland. Tel: +41 031631 5629; E-mail: ulrich.aschauer@dcb.unibe.ch 
like $\mathrm{LaZrO}_{2} \mathrm{~N}^{11}$ are considered fully disordered.

While understanding the $\mathrm{O} / \mathrm{N}$ arrangement in the bulk has been a major priority in the field of oxynitrides, little attention has been devoted to the chemical composition and anion ordering at surfaces. Since the topmost surface layers are in direct contact with the environment, we expect their composition and structure to significantly affect photo-catalytic processes and hence the water splitting efficiency of the oxynitride. A particularly well-studied perovskite oxynitride composition is $\mathrm{LaTiO}_{2} \mathrm{~N}$, which was experimentally shown to successfully evolve $\mathrm{O}_{2}$ and $\mathrm{H}_{2}$ in the presence of sacrificial agents ${ }^{12-14}$ and cocatalysts ${ }^{15}$. In the bulk, $\mathrm{LaTiO}_{2} \mathrm{~N}$ was reported to be either full disordered ${ }^{11,16}$ or to have partial ordering on the anion sub-lattice when the material is slowly cooled to room temperature, instead of being quenched ${ }^{17}$. Previous theoretical studies suggest a fully-ordered model with $\mathrm{N}$-atoms positioned in cis-manner ${ }^{16,18,19}$. Other studies considered only the trans-ordering without comparing it to other orderings ${ }^{20}$. Based on the formation energies of possible stoichiometric, oxygenrich and anion-deficient triclinic primitive cells, $\mathrm{LaTiO}_{2} \mathrm{~N}$ was shown to be made up of six aperiodic primitive cells ${ }^{19}$. The stoichiometric of these cells have cisorder and together with oxygen-rich cells form the surfaces of crystals, where those containing anion vacancies are found only in the bulk ${ }^{19}$.

By scanning tunnelling electron microscopy (STEM) combined with electron energy-loss spectroscopy (EELS), it was shown that the topmost layer of the $\mathrm{LaTiO}_{2} \mathrm{~N}$ (001) surface contains La ions ${ }^{21}$. After annealing the La layer at the surface is reconstructed and nitrogen deficient, but an aqua regia treatment restores the perfect geometry and stoichiometry, leading to a much improved photocatalytic activity ${ }^{21}$. $\mathrm{LaTiO}_{2} \mathrm{~N}$ thin films were found to most commonly expose (001) orientated surfaces ${ }^{22,23}$ but it was also shown that different substrates promote other surface orientations with distinctively different photocatalytic activities ${ }^{24}$.

These experimental findings support the assumption that the surface structure and composition will have a significant effect on the photocatalytic activity - nevertheless very little is know about the atomic arrangement at the surface. Existing theoretical work considered only the $\mathrm{TiO}_{2}$ termination of the (001) surface on top of a trans-type $\mathrm{TiO}_{2} / \mathrm{LaN}$ stacking, without considering other anion orderings at the surface or in the bulk ${ }^{25}$. In the present work, we aim to provide insights on the surface structure and composition via density functional theory (DFT) calculations and to establish if there is a relation between the anion ordering in the bulk and at the (001) surface of $\mathrm{LaTiO}_{2} \mathrm{~N}$. Moreover we want to determine how changes in ordering affect the physicochemical properties of the surface. Our results for a single surface of relative structural simplicity, allow us to rationalise the findings in terms of fundamental chemical and electrostatic interactions, which will form the basis for a more general understanding of anion ordering effects at perovskite oxynitride surfaces. We start our study by revisiting the anion order in the bulk and then use the various bulk models as starting point for surface slab calculations. Our results show that the bulk and the surface prefer different anion orderings, the latter being driven by electrostatic requirements to cancel surface dipoles rather than purely chemical effects. 
We find that in contact with vacuum, the most stable (001) surface is LaN terminated and that higher energy termination will have a tendency to reconstruct to avoid unfavourable surface dipoles.

\section{Computational methods}

Our density functional theory (DFT) calculations are performed with the Quantum ESPRESSO package ${ }^{26}$, using the PBE functional ${ }^{27}$. Ultrasoft pseudopotentials $^{28}$ are used for all elements and wavefunctions are expanded in plane waves with a kinetic-energy cut-off of 40 Ry and a cut-off of 320 Ry for the augmented density. A Hubbard $\mathrm{U}^{29}$ of $3.0 \mathrm{eV}$ is applied on the Ti $3 d$ orbitals.

We use the orthorhombic cell with space group P $\overline{1} / \mathrm{I} \overline{1}$, as refined by Clarke et al. ${ }^{11}$ as the starting point for our bulk geometry optimisations. As a result of the octahedral rotations $\left(\mathrm{a}^{-} \mathrm{b}^{-} \mathrm{c}^{-}\right.$Glazer pattern $\left.{ }^{30}\right)$, calculations based on this structure have more structural degrees of freedom compared to previous simulations based on a $\left(\mathrm{a}^{0} \mathrm{~b}^{-} \mathrm{b}^{-}\right)$cell with Imma space group ${ }^{16}$. In contrast to other theoretical studies ${ }^{18}$, both ionic positions and cell parameters are relaxed during geometry optimisation. Convergence thresholds of $1.4 \cdot 10^{-5} \mathrm{eV}$ for the energy and $0.05 \mathrm{eVA}^{-1}$ for the forces are used for all relaxations.

The surface models used in this study are asymmetric, stoichiometric and electroneutral (001) slabs with 4 unit-cell layers along the surface normal. The atoms in the bottom unit-cell layer are kept fixed at bulk positions. In order to eliminate spurious interactions between periodic images along the surface normal and to compensate the depolarising field resulting from the asymmetry of the slab, $10 \AA$ of vacuum is added and a dipole correction is used for all slab calculations ${ }^{31}$. The strength of the depolarising field that appears as a result of the chemical composition of the atomic layers and the one-sided relaxation of the slab, is in the most extreme case half the convergence threshold for the forces and its effect on the relaxed slab geometries is thus not significant ${ }^{32}$.

Monkhorst-Pack k-point meshes ${ }^{33}$ with dimensions $6 \times 4 \times 6$ and $6 \times 6 \times 1$ were chosen for the bulk and the surfaces respectively. VESTA ${ }^{34}$ has been used for the graphical presentation of all optimised structures. Electron and hole effective masses are computed using the finite differences method implemented in the program EMC. ${ }^{35}$

\section{Results \& Discussion}

\subsection{Bulk ordering and electronic structure}

Calculations were performed for all possible non-equivalent anion orderings in the 20-atom unit cells. The most relevant structures are presented in Figure 1, whereas others can be found in Figure $\mathrm{S} 1 \dagger$ with energies given in Table S1†. We classify the ordering in terms of Ti-N-Ti bonds lying either along one crystal axis (i.e. trans-chains, in models 1 and 2), or cis-chains meandering in one plane at $90^{\circ}$ angles (in models 3 and 4). Arrangements of these Ti-N-Ti chains along different directions are non-equivalent due to different degrees of octahedral rotations in the structure (compare model 1 and 2 as well as model 3 and 4, 

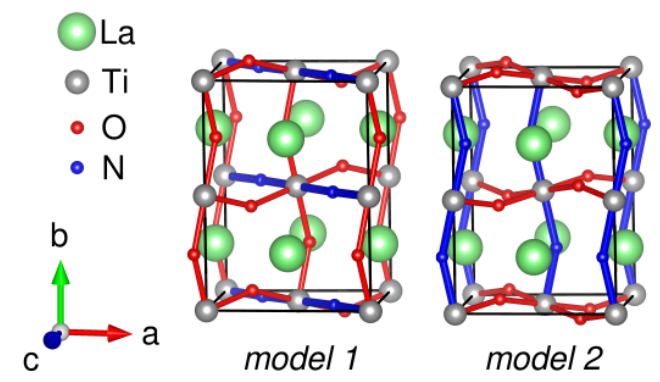

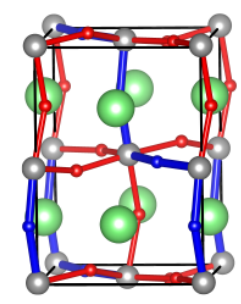

model 3

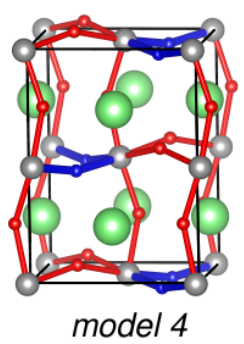

Figure 1: Four representative $\mathrm{O} / \mathrm{N}$ arrangements in the bulk cell. Other structures can be found in the Figure $\mathrm{S} 1 \dagger$

Table 1: Comparison between the most stable models of $\mathrm{LaTiO}_{2} \mathrm{~N}$ in bulk in terms of relative energy difference per formula unit with respect to the most stable models after geometry optimization. We also report band gaps and space groups for the models, where the tolerance threshold for the space group determination is $10^{-4} \AA$

\begin{tabular}{llll}
\hline Model & Relative energy $(\mathrm{eV})$ & Band gap $(\mathrm{eV})$ & Space group \\
\hline model 1 & 0.146 & 1.17 & $\mathrm{P} \overline{1}$ \\
model 2 & 0.311 & 1.16 & $\mathrm{P} \overline{1}$ \\
model 3 & 0.000 & 1.43 & $\mathrm{P} 1$ \\
model 4 & 0.000 & 1.43 & $\mathrm{P} 1$ \\
\hline
\end{tabular}

respectively).

We determined the energetically most favourable $\mathrm{O} / \mathrm{N}$ ordering to consist of $2 \mathrm{D}$ cis-chains (models 3 and 4), in agreement with other studies on $\mathrm{LaTiO}_{2} \mathrm{~N}^{16,18,19}$ and similar systems 7,36 . The two models are indistinguishable in terms of their energy (see Table 1) and relax to very similar geometries, irrespective of the initially different octahedral rotation angles. We note that these orderings lower the symmetry of the system to space group P1, as opposed to Pi in experiment and changes the Glazer pattern to $\mathrm{a}^{-} \mathrm{b}^{+} \mathrm{a}^{-}$and $\mathrm{a}^{-} \mathrm{a}^{+} \mathrm{b}^{-}$respectively (see table S $2 \dagger$ ).

A linear arrangement of the Ti-N-Ti bonds, on the other hand, (models 1 and 2 ) is found to be higher in energy. This stems from the fact that contrary to the zigzag ordering, it does not benefit from forming stronger bonds via the most favourable cis interaction between the Ti-3d and the N-2p orbitals ${ }^{37}$. Indeed, as shown in Table S2 $\dagger$, both models have longer Ti-N bonds compared to the zigzag arrangements. Moreover, the initial octahedral rotation appears to have a strong influence on the final optimised structure and we find two distinct geometries, both of which retain the experimental Pi symmetry.

As shown in the Tables S2 and S3†, the lattice parameters, bond lengths and octahedral rotation angles of all models are - where directly comparable - within $1 \%$ with respect to experiment. This highlights the small magnitude of geometry 
changes that occur when considering full anion order in our calculations and also shows the validity of our computational approach.

The anion arrangement also affects the electronic structure of the material, as shown for other oxynitrides ${ }^{38}$. In oxynitrides, the top of the valence band consists mainly of $\mathrm{N} 2 \mathrm{p}$ states with the $\mathrm{O} 2 \mathrm{p}$ states at lower energies (see Figure 2 ). Indeed, the cis-chain models (models 3 and 4) have a characteristic small shoulder of only nitrogen at the top of the valence band, which is absent for the linear arrangements (models 1 and 2). The latter have a large group of peaks, where both $\mathrm{N}$ and $\mathrm{O}$ states can be found. Interestingly enough, however, model 2 is the only one of the four orderings to have a direct $(\Gamma-\Gamma)$ band gap, an optimal condition for photoabsorption, and not an indirect one as the others (see Figure 2). Moreover its band gap is lower with respect to the cis-chains by about $0.27 \mathrm{eV}$, as shown in Table 1 . We want to note however that the bands leading to indirect gaps are very flat, which will on one hand diminish the effect of the indirect gap and on the other hand means that small structural or computational changes can affect this result. Our semi-local DFT calculations inevitably predict too small band gaps with respect to the experimental value of $2.1 \mathrm{eV}^{12}$. Calculations for selected models with the hybrid functional HSE06 ${ }^{39}$ $(x=0.18)$ give band gaps of 1.89 and $2.37 \mathrm{eV}$ (see Table $\mathrm{S} 1 \dagger)$ and agree well with experiment.

The calculated effective masses (see Table 2) of the electrons and holes are quite anisotropic, more strongly so for the cis-chains orderings. All models, however, have a light hole in at least one direction, which implies good hole mobility. Model 2 has the lowest average hole effective mass, which stems from the absence of flat N 2p bands at the top of the valence band as observed for all other orderings. Previously computed average electron and hole effective masses of 0.5 and $-0.9 \mathrm{~m}_{0}$ respectively, for cubic $\mathrm{LaTiO}_{2} \mathrm{~N}$ compare well with our results ${ }^{5}$. The difference in absolute values can be attributed to the octahedral rotations that we consider in the present study.

\subsection{Surfaces}

We investigate the structure and anion arrangement on the $\mathrm{LaTiO}_{2} \mathrm{~N}(001)$ surface using asymmetric slabs, which are both stoichiometric and electro-neutral. In the case of $\mathrm{ABX}_{3}(\mathrm{X}=\mathrm{O}, \mathrm{N})$ perovskite (001) surfaces there are two possible terminations, one having an $\mathrm{AX}$ and one having a $\mathrm{BX}_{2}$ composition ${ }^{40-43}$. An asymmetric slab inevitably exposes one of these terminations at the top surface and the other one at the bottom surface. In order to nevertheless calculate the surface energies of the individual terminations, we follow the procedure suggested by Eglitis and Vanderbilt ${ }^{42}$. The cleavage energy $\mathrm{E}_{\text {cleave, }}$, which is the energy required to break the bonds in the crystal when creating a surface (see Eq. 1), is the same for both terminations. When relaxing the slab, we keep the atoms at the bottom of the slab fixed to their bulk positions. The relaxation energy $\mathrm{E}_{\text {relax }}$ of the bottom termination is therefore zero while the one for the top termination is given by Eq. 2. Based on these considerations, we can determine the surface energy of the top termination as given by Eq. 3, where $E_{\text {slab }}^{\text {unrelaxed }}$ is the energy of the slab before relaxation, $E_{\text {bulk }}$ is the energy of the bulk, $E_{\text {slab }}^{\text {relaxed }}$ 


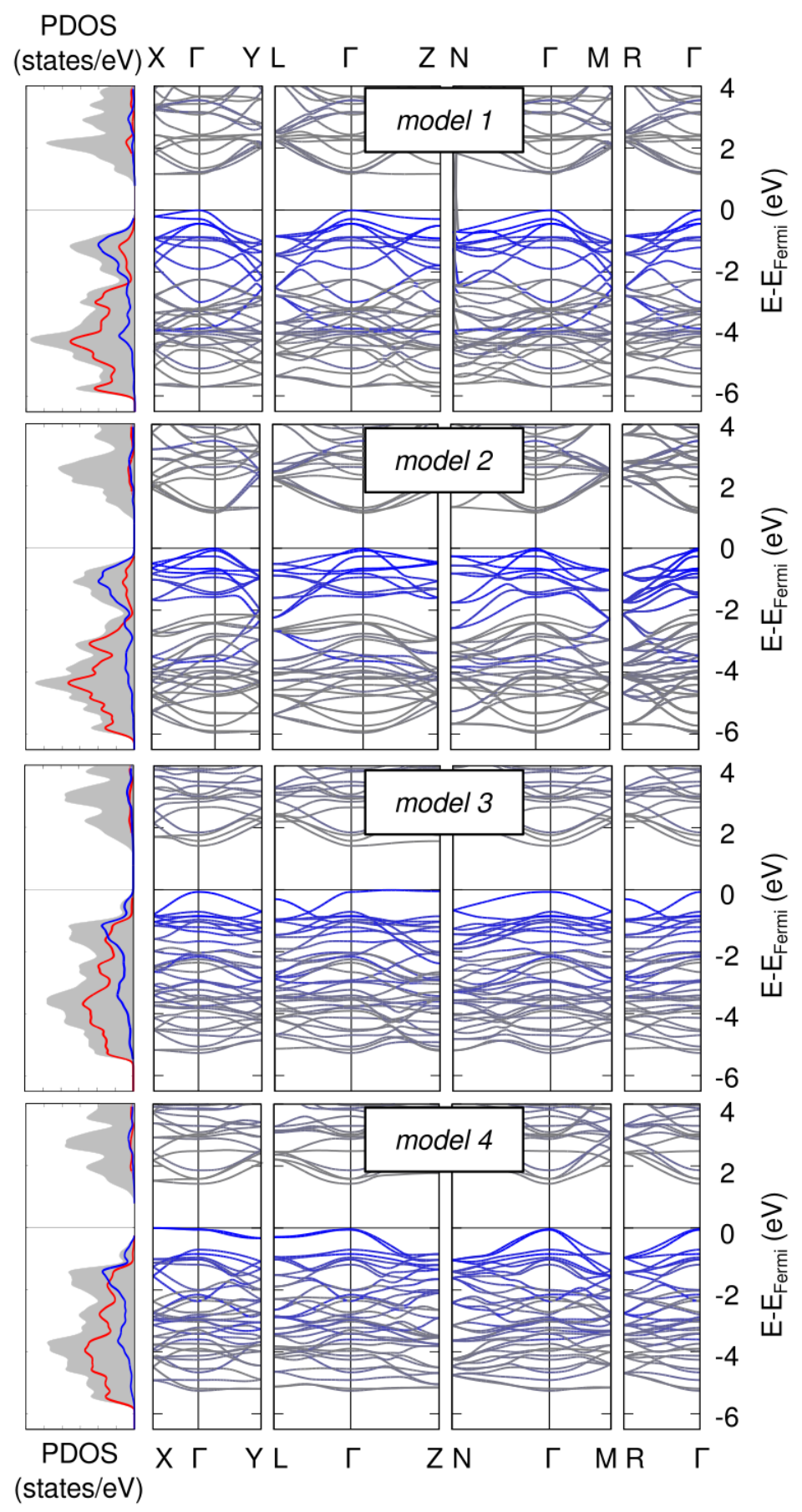

Figure 2: Projected density of states (PDOS) and bands for each bulk model. The $\mathrm{N}-2 \mathrm{p}$ states are in blue and the $\mathrm{q}-2 \mathrm{p}$ in red. A Gaussian broadening of 0.01 Ry is used for the PDOS. The nitrogen contribution to the different bands is shown as blue fat bands. Coordinates of the points in reciprocal space are given in Table $\mathrm{S} 5 \dagger$ 
Table 2: Hole effective masses (in unit of $\mathrm{m}_{0}$ electron rest mass) of the four models with the principal directions in fractional coordinates of their corresponding bulk cell (see Table S $3 \dagger$ )

\begin{tabular}{|c|c|c|c|}
\hline \multicolumn{4}{|c|}{ Hole effective masses } \\
\hline model 1 & & model & \\
\hline-0.17 & $(1.000 .000 .96)$ & -0.26 & 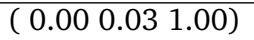 \\
\hline-1.66 & $(-0.960 .001 .00)$ & -0.29 & ( $0.001 .00-0.05)$ \\
\hline-1.70 & $\left(\begin{array}{llll}-0.00 & 1.00 & 1.00\end{array}\right)$ & -0.97 & $\left(\begin{array}{lll}1.00 & 0.00 & 0.00)\end{array}\right.$ \\
\hline model 3 & & model & \\
\hline-0.33 & $\left(\begin{array}{lll}1.00 & -0.98 & 0.99)\end{array}\right.$ & -0.25 & $(0.000 .001 .00)$ \\
\hline-0.95 & ( 1.001 .000 .95$)$ & -1.05 & ( 1.000 .000 .00$)$ \\
\hline-5.45 & $(-0.970 .011 .00)$ & -4.54 & $(0.001 .00-0.00)$ \\
\hline \multicolumn{4}{|c|}{ Electron effective masses } \\
\hline model 1 & & model & \\
\hline$\infty$ & 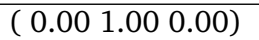 & 0.91 & 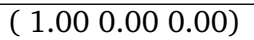 \\
\hline 0.57 & $(-0.940 .001 .00)$ & 0.80 & ( 0.000 .001 .00$)$ \\
\hline 0.35 & ( 1.000 .000 .93$)$ & 0.25 & $(0.001 .000 .00)$ \\
\hline model 3 & & model & \\
\hline 3.93 & $(-0.830 .061 .00)$ & 3.40 & $(0.001 .000 .00)$ \\
\hline 2.51 & ( 1.000 .880 .71$)$ & 0.72 & ( 1.000 .000 .00$)$ \\
\hline 0.47 & ( $1.00-0.950 .95)$ & 0.32 & ( 0.000 .001 .00$)$ \\
\hline
\end{tabular}

is the energy of the slab after relaxation and $A_{\text {surf }}$ is the surface area of the slab.

$$
\begin{aligned}
E_{\text {cleave }} & =\frac{1}{2}\left(E_{\text {slab }}^{\text {unrelaxed }}-E_{\text {bulk }}\right) \\
E_{\text {relax }} & =E_{\text {slabed }}^{\text {rlaxed }}-E_{\text {slab }}^{\text {unrelaxed }} \\
E_{\text {surf }}^{\text {top }} & =\frac{E_{\text {cleave }}+E_{\text {relax }}}{A_{\text {surf }}} \\
& =\frac{2 E_{\text {slab }}^{\text {relaxd }}-E_{\text {slab }}^{\text {unrelaxed }}-E_{\text {bulk }}}{2 A_{\text {surf }}}
\end{aligned}
$$

Surfaces of oxynitrides are more complex to describe than the ones of pure oxides since the chemical composition of each single atomic layers can also vary, depending on the anion ordering. The linear Ti-N-Ti chains in models 1 and 2 can, for example, result in two extreme cases of either alternating $\left(\mathrm{La}_{2} \mathrm{O}_{2}\right)$ $\left(\mathrm{Ti}_{2} \mathrm{O}_{2} \mathrm{~N}_{2}\right)$ or $\left(\mathrm{La}_{2} \mathrm{~N}_{2}\right)-\left(\mathrm{Ti}_{2} \mathrm{O}_{4}\right)$ planes, depending on the cleavage direction. In addition, the cis-chains propagating along $\mathrm{z}$ (model 3), give rise to yet another sequence of layers: $\left(\mathrm{La}_{2} \mathrm{NO}\right)-\left(\mathrm{Ti}_{2} \mathrm{O}_{3} \mathrm{~N}\right)$. In order to cover the possible terminations in terms of anion ordering and octahedral rotation patterns, all models in Figure 1 were used as a starting point for the construction of slabs.

In Table 3 we report the surface energies computed for different possible terminations for a given bulk ordering and in Figure 3 we show some representative slab geometries. Interestingly, the models with the lowest surface energy are those originating from model 2, which had the highest energy in the bulk. 


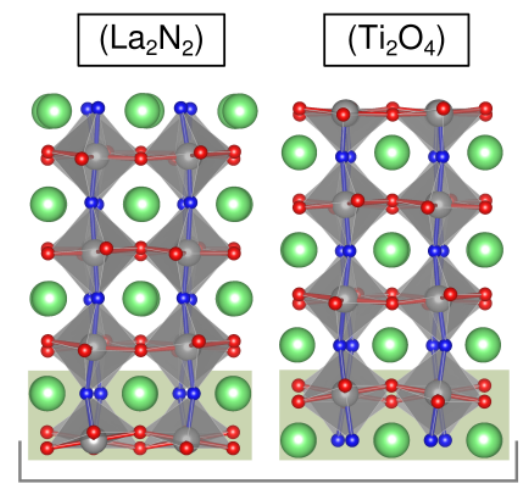

model 2 (010)

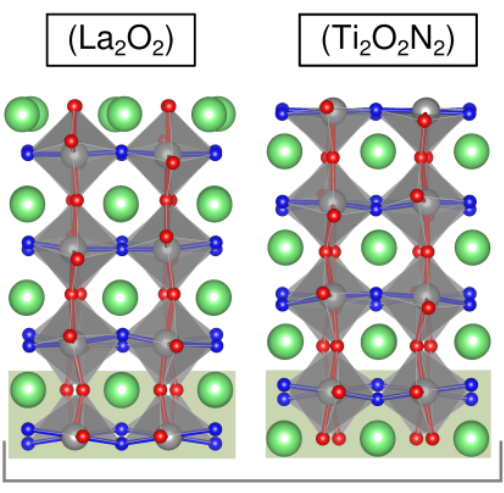

model 2 (001)

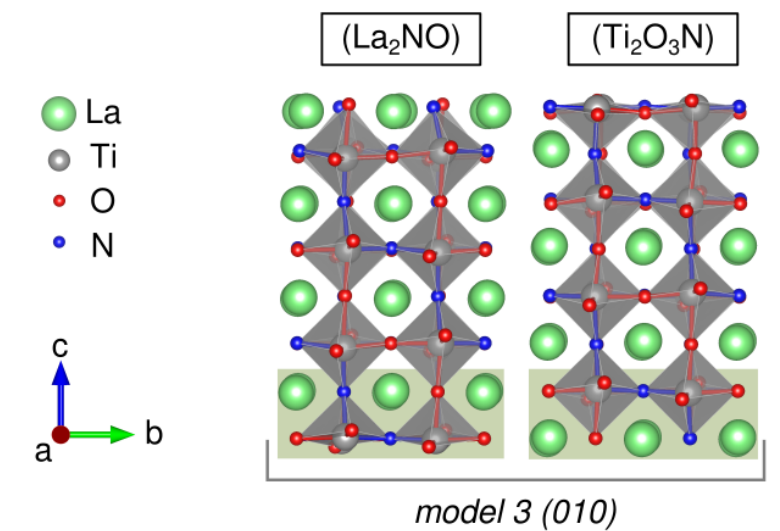

Figure 3: Optimised geometry of some representative slabs. The bottom unit cell, highlighted in light green, is kept fixed at bulk positions. Other structures can be found in Figure S2 $\dagger$ 
Table 3: Surface energies of the model surfaces of $\mathrm{LaTiO}_{2} \mathrm{~N}$ (001) with different termination layers and with octahedral rotations included

\begin{tabular}{lll}
\hline Model & Termination & $E_{\text {surf }}^{\text {top }}\left(\mathrm{J} / \mathrm{m}^{2}\right)$ \\
\hline model 1 & $\left(\mathrm{La}_{2} \mathrm{O}_{2}\right)$ & 1.309 \\
& $\left(\mathrm{Ti}_{2} \mathrm{O}_{2} \mathrm{~N}_{2}\right)$ & 1.448 \\
& $\left(\mathrm{La}_{2} \mathrm{~N}_{2}\right)$ & 1.226 \\
& $\left(\mathrm{Ti}_{2} \mathrm{O}_{4}\right)$ & 0.968 \\
\hline model 2 & $\left(\mathrm{La}_{2} \mathrm{O}_{2}\right)$ & 0.980 \\
& $\left(\mathrm{Ti}_{2} \mathrm{O}_{2} \mathrm{~N}_{2}\right)$ & 1.088 \\
& $\left(\mathrm{La}_{2} \mathrm{~N}_{2}\right)$ & 0.542 \\
& $\left(\mathrm{Ti}_{2} \mathrm{O}_{4}\right)$ & 0.654 \\
\hline model 3 & $\left(\mathrm{La}_{2} \mathrm{NO}\right)$ & 1.152 \\
& $\left(\mathrm{Ti}_{2} \mathrm{O}_{3} \mathrm{~N}\right)$ & 1.132 \\
\hline model 4 & $\left(\mathrm{La}_{2} \mathrm{O}_{2}\right)-\mathrm{N}$ & 1.249 \\
& $\left(\mathrm{Ti}_{2} \mathrm{O}_{2} \mathrm{~N}_{2}\right)-\mathrm{N}$ & 1.440 \\
& $\left(\mathrm{La}_{2} \mathrm{O}_{2}\right)-\mathrm{O}$ & 1.562 \\
& $\left(\mathrm{Ti}_{2} \mathrm{O}_{2} \mathrm{~N}_{2}\right)-\mathrm{O}$ & 1.535 \\
\hline
\end{tabular}

This can be related to the fact that a less favourable (higher energy) bulk phase will have a smaller cleavage energy compared to a very stable bulk phase. Similarly low surface energies are found for model 1, which is the other high-energy bulk ordering. We also want to note that for the surface of a nanoparticle or thin film, there will be an interface between the preferential anion ordering in the bulk and at the surface, which we do not consider in the present work. Such interfaces between bulk cis-ordering and surface trans-ordering are however relevant and will be the subject of further work.

The surface energy is also proportional to the dipole moment at the surface and electro-neutral atomic layers will lead to energetically more favourable surfaces. Indeed, both for model 1 and 2, the most stable surfaces consist of sequences of formally electro-neutral layers, $\left(\mathrm{La}_{2} \mathrm{~N}_{2}\right)-\left(\mathrm{Ti}_{2} \mathrm{O}_{4}\right)$. On the contrary, slabs with $\left(\mathrm{La}_{2} \mathrm{O}_{2}\right)-\left(\mathrm{Ti}_{2} \mathrm{O}_{2} \mathrm{~N}_{2}\right)$ sequences have a higher surface energy because the nominal charges of $2+$ and 2- on La- and Ti-layers respectively result in polar slabs. Such slabs will undergo electronic or structural reconstruction in order to compensate polarity ${ }^{44}$ as we can clearly see in the PDOS shown in Figure 4. The more oxygen is added to the La-layer, the more self-doping of the surface with electrons takes place, filling mainly the empty Ti-3d states. The same is true for the Ti-termination, where the model with the highest nitrogen content undergoes self doping with holes in order to compensate polarity.

As reported in Table S6†, the surface geometry (interplanar distances and surface rumplings) also changes to reduce the surface dipole. We can see for example that while all slabs undergo an inwards relaxation of the topmost layer, as characterised by a negative change of the interplanar spacing $\Delta_{1-2}$, this change is much weaker $(-2.5 \%)$ for the charge-neutral $\left(\mathrm{La}_{2} \mathrm{~N}_{2}\right)$ termination compared to the nominally polar $\left(\mathrm{La}_{2} \mathrm{O}_{2}\right)$ termination $(-7.9 \%)$ of model 1 . The resulting decreased interlayer spacing contributes to reducing the surface dipole. The 

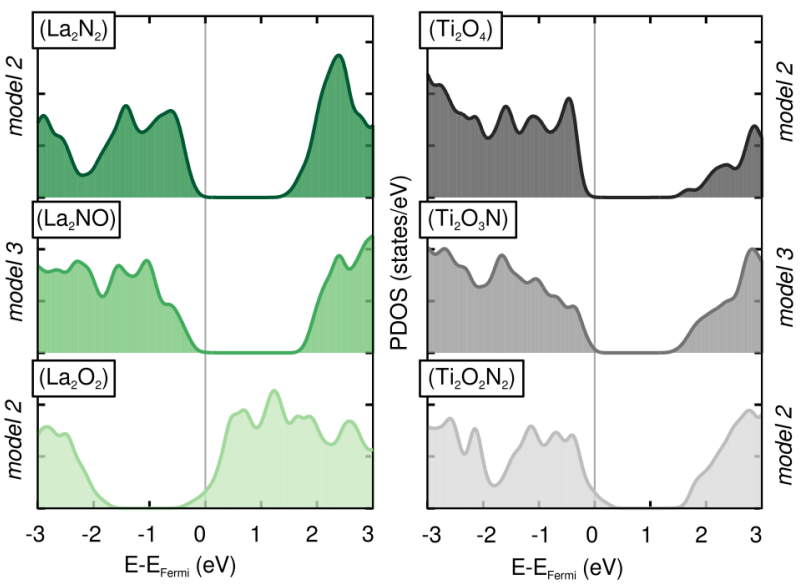

Figure 4: PDOS on the atoms of the topmost unit-cell with La- (left) and Titermination (right) of the surface models shown in Figure 3

geometry of nominally polar surfaces moreover exhibits much larger oxygen rumpling as seen for example in the case of the model $1\left(\mathrm{Ti}_{2} \mathrm{O}_{4}\right)$ and $\left(\mathrm{Ti}_{2} \mathrm{O}_{2} \mathrm{~N}_{2}\right)$ terminations, where the rumpling $\left(s_{O}\right)$ changes from $0.080 \AA$ to $-0.350 / 0.350$ $\AA$ respectively. In the case of cis-chains leading to mixed anion layers, we want to highlight the situation where octahedral rotations lead to structures where nitrogen and oxygen atoms form two separate planes, either above or below the titanium ones as shown in Figure $\mathrm{S} 2 \dagger$ for the $\left(\mathrm{La}_{2} \mathrm{O}_{2}\right)$ termination. This splitting into sub-planes has a strong effect on the surface energy, which for these terminations differs by $0.313 \mathrm{~J} / \mathrm{m}^{2}$.

As for the chemical composition of the topmost layer in contact with vacuum, our calculations predict the LaN-termination to be the most favourable. This is supported by experimental studies that found an increased amount of La in the topmost surface layers ${ }^{21,24}$.

\subsection{Structural reconstructions}

In addition to the aforementioned electronic reconstructions, structural reconstructions can occur for polar surfaces. We have tested three cases of structural reconstruction on three slabs with different degree of polarity and surface energies (model 2: $\left(\mathrm{La}_{2} \mathrm{O}_{2}\right)$, model 2: $\left(\mathrm{La}_{2} \mathrm{~N}_{2}\right)$, model 4: $\left.\left(\mathrm{La}_{2} \mathrm{O}_{2}\right)-\mathrm{O}\right)$, so as to estimate the effect they have on the surface stability.

We choose the three reconstruction models, schematically shown in Figure S3t, which are based on the work of Deacon-Smith et al. ${ }^{45}$ and which result in different La-anion bond patterns at the surface. In the first reconstruction, a square formed of La-X bonds, is called island; the second one keeps only the diagonal atoms and the third one creates trenches along the $a$ or $b$ axis. For all of these reconstructions, half of the atoms in the topmost atomic layer are moved to their bulk positions at the bottom of the slab and fixed, so as to maintain the 
Table 4: Surface energies of models with different geometric reconstructions

\begin{tabular}{llll}
\hline Model & Termination & Reconstruction & $E_{\text {surf }}^{\text {top }}\left(\mathrm{J} / \mathrm{m}^{2}\right)$ \\
\hline model 2 & $\left(\mathrm{La}_{2} \mathrm{~N}_{2}\right)$ & - & 0.542 \\
& & diagonal & 1.247 \\
& & island & 0.714 \\
& & trench-a & 0.881 \\
& & trench-b & 0.882 \\
\hline model 2 & - & 0.980 \\
& $\left(\mathrm{La}_{2} \mathrm{O}_{2}\right)$ & island & 1.387 \\
& & trench-a & 0.824 \\
& & trench-b & 0.895 \\
\hline model 4 & $\left(\mathrm{La}_{2} \mathrm{O}_{2}\right)-\mathrm{O}$ & - & 1.562 \\
& & diagonal & 1.745 \\
& & island & 1.027 \\
& & trench-a & 1.060 \\
& & trench-b & 1.060 \\
\hline
\end{tabular}

stoichiometry of the slab.

As shown in Table 4, any structural reconstruction on model 2: $\left(\mathrm{La}_{2} \mathrm{~N}_{2}\right)$ will lead to an increase in surface energy. This is expected because the surface is non-polar to start with and any geometric change will lead to less favourably coordinated ions at the surface. For both polar $\left(\mathrm{La}_{2} \mathrm{O}_{2}\right)$ slabs, however, we observe a lowering of the surface energy at least for some of the reconstructions.

Model 4: $\left(\mathrm{La}_{2} \mathrm{O}_{2}\right)-\mathrm{O}$, which without reconstructions has a fairly high surface energy of $1.562 \mathrm{~J} / \mathrm{m}^{2}$, can gain $0.535 \mathrm{~J} / \mathrm{m}^{2}$ with the island reconstruction (see Figure 5), as a result of strong geometry changes during relaxation. An oxygen atom moves from the underlying $\left(\mathrm{Ti}_{2} \mathrm{O}_{2} \mathrm{~N}_{2}\right)$ layer to the topmost one. Such a migration serves as a way to add electrons to the positively-charged $\mathrm{LaO}$ atomic layer. At the same time, the net charge of the Ti-layer is reduced, which guarantees a smooth transition from the surface to the bulk region. In this configuration, the surface is stabilised via the geometric reconstruction, while the electronic one is completely absent (see Figure 6). In addition, the relaxation of the trench-a model 4 results in the same geometry as the island and trench-b ones, which confirms the stability of such a structure.

Model 2: $\left(\mathrm{La}_{2} \mathrm{O}_{2}\right)$ is already more stable to start with than model 4: $\left(\mathrm{La}_{2} \mathrm{O}_{2}\right)$ $\mathrm{O}$ and thus, less energy can be gained via reconstructions. The biggest change is from 0.980 to $0.824 \mathrm{~J} / \mathrm{m}^{2}$ for the trench-a reconstruction (see Figure 6), which places the Fermi energy in the band gap, the surface thus being no longer electronically reconstructed. This configuration also undergoes strong geometry changes with two $\mathrm{N}$ atoms moving from the Ti-layer into the topmost La-layer. This situation, where $\mathrm{Ti}$ atoms become five-coordinated is more stable, highlighting the strong preference of $\mathrm{N}$ atoms to stay in the surface La layer. 


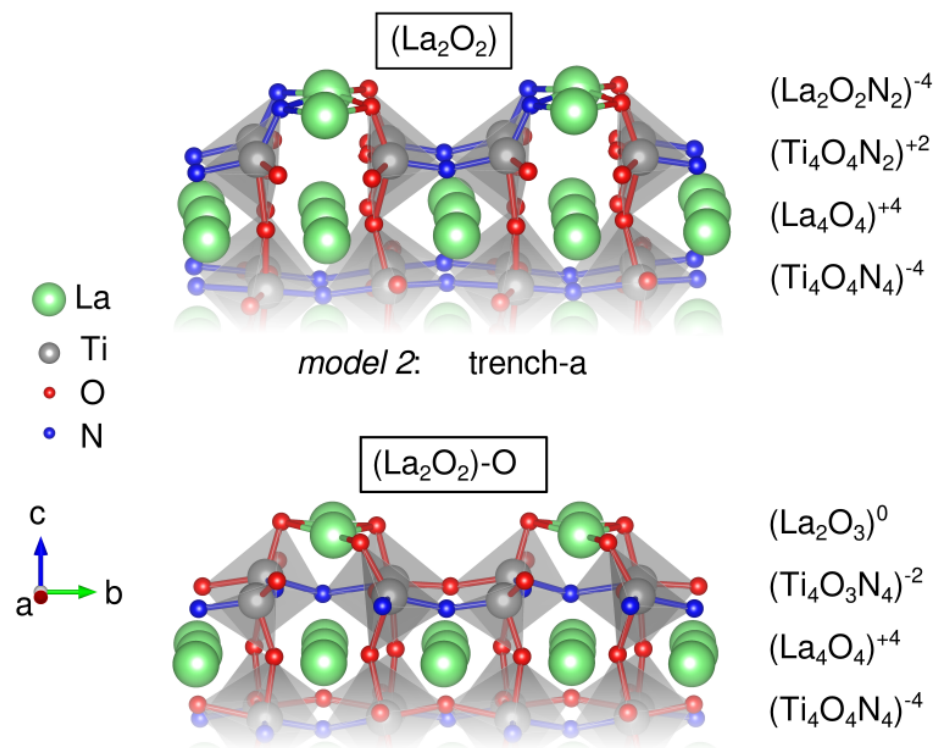

model 4: island

Figure 5: Geometry of two relaxed reconstructed models with doubled cell along $\mathrm{b}$, in order to improve the visualization of the reconstructions. The chemical composition for each layer is presented together with its formal charge. Other models can be found in Figure S4†.

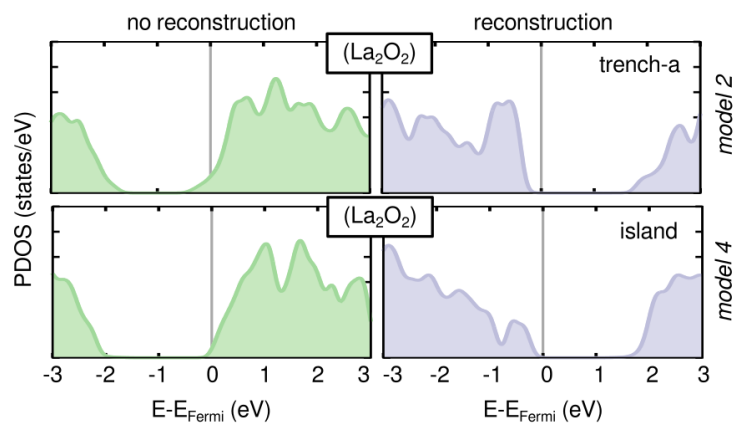

Figure 6: PDOS for two models, where the reconstruction leads to different electronic structure. The topmost layer comprises the reconstructed layer plus the underlying atomic layer 


\section{Conclusions}

We show that the preferential $\mathrm{O} / \mathrm{N}$ arrangement in the bulk and at (001) surfaces of the perovskite oxynitride $\mathrm{LaTiO}_{2} \mathrm{~N}$ is different. In the bulk $2 \mathrm{D}$ cis-chains of $\mathrm{N}$ atoms are preferred, in agreement with previous work and observations for similar compounds. The surface order is governed instead by a complex interplay between factors, such as chemical composition and degree of polarity. The most energetically favourable surface model is the La-terminated surface with alternating polar-compensated atomic layers. Other less stable surfaces get stabilised by undergoing both electronic and structural reconstructions. Our analysis of the driving forces governing anion ordering at the $\mathrm{LaTiO}_{2} \mathrm{~N}$ surface form the basis for understanding the surface composition and hence chemistry of other surfaces commonly exposed on nanoparticles and thin films of this technologically promising material.

\section{Acknowledgements}

This research was funded by the SNF Professorship Grant PP00P2 157615. Calculations were performed on UBELIX (http://www.id.unibe.ch/hpc), the HPC cluster at the University of Bern.

\section{References}

[1] S. G. Ebbinghaus, H. P. Abicht, R. Dronskowski, T. Müller, A. Reller and A. Weidenkaff, Progress in Solid State Chemistry, 2009, 37, 173-205.

[2] T. Takata, C. Pan and K. Domen, Science and Technology of Advanced Materials, 2015, 16, 033506.

[3] M. Ahmed and G. Xinxin, Inorganic Chemistry Frontiers, 2016, 3, 578-590.

[4] Y. Wu, P. Lazic, G. Hautier, K. Persson and G. Ceder, Energy Environmental Science, 2013, 6, 157-168.

[5] I. E. Castelli, D. D. Landis, K. S. Thygesen, S. Dahl, I. Chorkendorff, T. F. Jaramillo and K. W. Jacobsen, Energy \& Environmental Science, 2012, 5, 9034.

[6] A. Fuertes, Dalton Transactions, 2010, 39, 5942-48.

[7] J. P. Attfield, Crystal Growth and Design, 2013, 13, 4623-4629.

[8] A. Fuertes, Inorganic Chemistry, 2006, 45, 9640-9642.

[9] M. Yang, J. Oró-Solé, J. A. Rodgers, A. B. Jorge, A. Fuertes and J. P. Attfield, Nature Chemistry, 2011, 3, 47-52.

[10] K. Page, M. W. Stoltzfus, Y.-I. Kim, T. Proffen, P. M. Woodward, A. K. Cheetham and R. Seshadri, Chemistry of Materials, 2007, 19, 4037-4042. 
[11] S. J. Clarke, B. P. Guinot, C. W. Michie, M. J. C. Calmont and M. J. Rosseinsky, Chemistry of Materials, 2002, 14, 288-294.

[12] A. Kasahara, K. Nukumizu, G. Hitoki, T. Takata, J. N. Kondo, M. Hara, H. Kobayashi and K. Domen, The Journal of Physical Chemistry A, 2002, 106, 6750-6753.

[13] A. E. Maegli, E. H. Otal, T. Hisatomi, S. Yoon, C. M. Leroy, N. Schäuble, Y. Lu, M. Grätzel and A. Weidenkaff, Energy Procedia, 2012, 22, 61-66.

[14] J. Feng, W. Luo, T. Fang, H. Lv, Z. Wang, J. Gao, W. Liu, T. Yu, Z. Li and Z. Zou, Advanced Functional Materials, 2014, 24, 3535-3542.

[15] A. E. Maegli, S. Pokrant, T. Hisatomi, M. Trottmann, K. Domen and A. Weidenkaff, The Journal of Physical Chemistry C, 2013, 118, 16344-16351.

[16] M. Yashima, M. Saito, H. Nakano, T. Takata, K. Ogisu and K. Domen, Chemical communications, 2010, 46, 4704-4706.

[17] D. Logvinovich, L. Bocher, D. Sheptyakov, R. Figi, S. G. Ebbinghaus, R. Aguiar, M. H. Aguirre, A. Reller and A. Weidenkaff, Solid State Sciences, 2009, 11, 1513-1519.

[18] A. Kasahara, K. Nukumizu, T. Takata, J. N. Kondo, M. Hara, H. Kobayashi and K. Domen, Journal of Physical Chemistry B, 2003, 107, 791-797.

[19] X. Wang, Z. Li and Z. Zou, Physical Chemistry Chemical Physics, 2015, 17, 19631-19636.

[20] N. Umezawa and A. Janotti, ChemSusChem, 2016, 9, 1027-1031.

[21] M. Matsukawa, R. Ishikawa, T. Hisatomi, Y. Moriya, N. Shibata, J. Kubota, Y. Ikuhara and K. Domen, Nano Letters, 2014, 14, 1038-1041.

[22] C. Le Paven-Thivet, L. Le Gendre, J. Le Castrec, F. Cheviré, F. Tessier and J. Pinel, Progress in Solid State Chemistry, 2007, 35, 299-308.

[23] A. Ziani, C. Le Paven-Thivet, L. Le Gendre, D. Fasquelle, J. C. Carru, F. Tessier and J. Pinel, Thin Solid Films, 2008, 517, 544-549.

[24] M. Pichler, W. Si, F. Haydous, H. Téllez, J. Druce, E. Fabbri, M. El Kazzi, M. Döbeli, S. Ninova, U. Aschauer, A. Wokaun, D. Pergolesi and T. Lippert, Advanced Functional Materials, in press.

[25] J. H. Montoya, M. Garcia-Mota, J. K. Nørskov and A. Vojvodic, Physical chemistry chemical physics : PCCP, 2015, 17, 2634-40.

[26] P. Giannozzi, S. Baroni, N. Bonini, M. Calandra, R. Car, C. Cavazzoni, D. Ceresoli, G. L. Chiarotti, M. Cococcioni, I. Dabo, A. Dal Corso, S. de Gironcoli, S. Fabris, G. Fratesi, R. Gebauer, U. Gerstmann, C. Gougoussis, A. Kokalj, M. Lazzeri, L. Martin-Samos, N. Marzari, 
F. Mauri, R. Mazzarello, S. Paolini, A. Pasquarello, L. Paulatto, C. Sbraccia, S. Scandolo, G. Sclauzero, A. P. Seitsonen, A. Smogunov, P. Umari and R. M. Wentzcovitch, Journal of Physics: Condensed Matter, 2009, 21, 395502.

[27] J. Perdew, K. Burke and M. Ernzerhof, Physical Review Letters, 1996, 77, 3865-3868.

[28] D. Vanderbilt, Physical Review B, 1990, 41, 7892-7895.

[29] V. I. Anisimov, J. Zaanen and O. K. Andersen, Physical Review B, 1991, 44, 943-954.

[30] A. M. Glazer, Acta Crystallographica Section B Structural Crystallography and Crystal Chemistry, 1972, 28, 3384-3392.

[31] L. Bengtsson, Physical Review B, 1999, 59, 12301-12304.

[32] C. Cheng, K. Kunc and M. H. Lee, Physical Review B, 2000, 62, 409-418.

[33] H. J. Monkhorst and J. D. Pack, Physical Review B, 1976, 13, 5188-5192.

[34] K. Momma and F. Izumi, Journal of Applied Crystallography, 2011, 44, $1272-1276$.

[35] A. Fornari and C. Sutton, Effective Mass Calculator.

[36] L. Clark, J. Oró-Solé, K. S. Knight, A. Fuertes and J. P. Attfield, Chemistry of Materials, 2013, 25, 5004-5011.

[37] K. Tatsumi and R. Hoffmann, Inorganic chemistry, 1980, 19, 2656-2658.

[38] A. Kubo, G. Giorgi and K. Yamashita, Chemistry of Materials, 2017, 29, 539-545.

[39] A. V. Krukau, O. A. Vydrov, A. F. Izmaylov and G. E. Scuseria, Journal of Chemical Physics, 2006, 125, 224106.

[40] S. Piskunov, E. Kotomin, E. Heifets, J. Maier, R. Eglitis and G. Borstel, Surface Science, 2005, 575, 75-88.

[41] E. Heifets, S. Piskunov, E. A. Kotomin, Y. F. Zhukovskii and D. E. Ellis, Physical Review B, 2007, 75, 115417.

[42] R. I. Eglitis and D. Vanderbilt, Physical Review B, 2007, 76, 155439.

[43] R. I. Eglitis and D. Vanderbilt, Physical Review B, 2008, 78, 1-11.

[44] J. Goniakowski, F. Finocchi and C. Noguera, Reports on Progress in Physics, 2008, 71, 016501.

[45] D. E. E. Deacon-Smith, D. O. Scanlon, C. R. A. Catlow, A. A. Sokol and S. M. Woodley, Advanced Materials, 2014, 26, 7252-7256. 